\title{
Innovative Breeding Methods to Enhance the Oleic Acid Content in Peanut for Better Oil Quality and Human Health
}

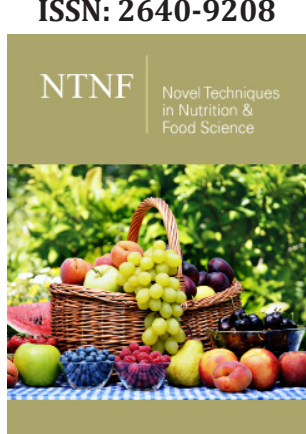

${ }^{* 1}$ Corresponding author: Hajisaheb LNf, Department of Genetics and Plant Breeding, India

Submission: 韭June 11, 2019

Published: 眥 March 19, 2020

Volume 4 - Issue 5

How to cite this article: Hajisaheb LNf, Ramesh SB, Kavera B, Mangesh PJ ,Malagoud Dp. Innovative Breeding Methods to Enhance the Oleic Acid Content in Peanut for Better Oil Quality and Human Health. Nov Tech Nutri Food Sci. 4(5).NTNF.000599.2020.

DOI: 10.31031/NTNF.2020.04.000599

Copyright@ Hajisaheb LNf. This article is distributed under the terms of the Creative Commons Attribution 4.0 International License, which permits unrestricted use and redistribution provided that the original author and source are credited.

\author{
Hajisaheb LNf ${ }^{1 *}$, Ramesh $\mathrm{SB}^{2}$, Kavera $\mathrm{B}^{1}$, Mangesh $\mathrm{PJ}^{2}$ and Malagoud $\mathrm{Dp}^{2}$ \\ ${ }^{1}$ Department of Genetics and Plant Breeding, India \\ ${ }^{2}$ Department of Biotechnology, India
}

Keywords: $19^{\text {th }}$ century; Prisoner nutrition; Rumford soup

\section{Mini Review}

Cultivated peanut is an important oilseed and legume crop. The quality of the peanut oil depends on its chemical properties especially the fatty acid composition. Oils rich in monounsaturated fatty acids have health benefits and enhanced oil quality such as better oil stability, flavor and nutrition. Oleic acid, a monounsaturated (C18:1) has cholesterollowering properties [1]. In addition, oleic acid helps to decrease the inflammation of the endothelial cells [2]. Dietary intake of oleic acid can also protect endothelial cells against hydrogen peroxide oxidative stress and thus reduce susceptibility of low-density lipoproteins to oxidative modifications [2]. Therefore, one of the major goals for peanut breeding is to increase the oleic acids and thereby enhancing the ratio of oleic acid to linoleic acid.

Although as many as 12 fatty acids have been reported in peanuts, only three (palmitic, oleic and linoleic) are present in amounts exceeding 5\% [3]. These three fatty acids comprise about $90 \%$ of the total fatty acid composition of the oil, with oleic (36-67\%) and linoleic (15$43 \%$ ) comprising about $80 \%$ depending on the variety [4]. It has been reported that Virginiatype peanuts naturally produce oil with slightly lower linoleic percentage and therefore have greater oil stability than Spanish and Valencia-types [5]. Accessions belonging to A hypogaea (Virginia-types) had higher oleic acid (54.16\%) compared with those of ssp. fastigiata (Spanish and Valencia) (45.70\%). An O/L ratio up to 6.93 was found among ssp. hypogaea. Among the Florida breeding lines, the oleic/linoleic (O/L) ratio ranged from 0.9 to $35: 1$ [6]. Among the ICRISAT mini core collection accessions [7], oleic acid ranged from $33.60 \%$ to $73.54 \%$. Additional varieties, mutants, germplasm lines and breeding lines had oleic acid within the range of mini core accessions [8,9] screened US mini core [10] for the point mutation (G-to-A at position 448 resulting in a D150N amino acid substitution) at ahFAD2A using CAPS marker and found mutant allele in $31.6 \%$ of the accessions.

A naturally occurring high-oleate spontaneous mutant, F435 was identified in the germplasm F78-1339 [6]. This high-oleate mutant produces the seed oil comprising of $80 \%$ oleate and as little as $2 \%$ linoleate [6]. One spontaneous (8-2122) and two chemically induced (Mycogen-Flavo and M2-225) mutants were developed from a normal oleate content line, AT-108 [11]. Subsequently, Sun Oleic 95R was developed from a breeding program involving F435 and F519-9. Sun Oleic 95R with 49\% oil and 80.6\% oleic acid was released in 1995 [12]. Sun Oleic 97R, an improved genotype over Sun Oleic 95R, derived from the backcrossing between F435-2-2-E-2-l-b4-E-b2-b3-l-E, a high oleic line and F519-9 (Sun runner, recurrent parent) was released in 1997. It recorded $48.5 \%$ oil and $81.8 \%$ oleic acid [13]. Microsomal oleoyl-PC (phosphatidyl choline) desaturase is responsible for the conversion of oleate to linoleate in non-photosynthetic tissues and developing seeds. Activity of microsomal oleoylPC desaturase is significantly lower in some high-oleate peanut seeds [14]. The high-oleate trait in peanut is due to a recessive mutation [4].

In the previous work from [15], two homologous oleoyl-PC desaturase genes (ahFAD2A and ahFAD2B), each having its origin in different diploid progenitor species, were isolated 
and characterized from the cultivated peanut. The expression patterns of both the genes were examined in peanut varieties with normal oleate and high-oleate phenotypes. Both the homologous genes expressed in normal oleate peanuts, whereas the expression of one gene (ahFAD2B) is severely reduced or absent in high-oleate mutants (8-2122 and M2-225). Also, there was a mutation in the ahFAD2A gene in normal oleate and high-oleate lines that encode a protein of reduced function [15]. Therefore, a significant reduction in the transcript levels of ahFAD2B and a mutation in ahFAD2A are responsible for the high-oleate phenotype in these peanut varieties, and the expression of one gene encoding a functional enzyme appears to be sufficient for the normal oleate/linoleate phenotype.

Further, a detailed examination was carried out at the coding regions and the regulatory elements of both these genes in the high-oleate mutants (8-2122, Mycogen-Flavo and M2-225) and the normal variety (AT-108). The two chemically induced, high-oleate mutants (Mycogen-Flavo and M2-225) showed an insertion of the miniature inverted repeat transposable elements (MITE) in the ahFAD2B gene at the base position 656 in the Mycogen-Flavo, and at 998 in M2-225. This MITE led to premature stops in both the mutants. In all mutant lines, the ahFAD2A gene contained a point mutation previously described by Jung et al. [15]. Studies on the putative promoter region of the ahFAD2A and ahFAD2B genes in both the normal and mutant lines did not reveal any significant changes. Interestingly, no differences were found at ahFAD2B gene of the 8-2122 mutant and that of the normal; hence it is difficult to explain the high-oleate phenotype of 8-2122. Wang et al. [16] reported six novel full-length cDNA sequences (named as AhFAD2-1, $-2,-3,-4,-5$, and -6 ) in peanut, The results suggested that there might be more candidate genes controlling levels of oleate in developing seeds and the presence of complex gene networks controlling the fluxes between the endoplasmic reticulum and the chloroplast within the peanut cells.

With this background knowledge on the genetic nature of the oleic acid content in peanut, various methods of breeding have been envisaged. Mutation breeding in GPBD 4, a high yielding and foliar disease resistant genotype, resulted in a high-oleate mutant Dh 245 (GM 4-3) [17] at UAS, Dharwad, India. Currently, marker-assisted backcrossing (MABC) and marker-assisted selection (MAS) are employed since the gene-specific and allele-specific DNA markers are available $[9,18,19]$. Two FAD2 mutant alleles from Sun Oleic 95R were transferred into the genetic background of elite varieties such as ICGV 06110, ICGV 06142 and ICGV 06420. The oleate content varied from 62 to $83 \%$ among the backcross lines. Oleic acid increased by 0.5-1.1 folds, with concomitant reduction of linoleic acid by 0.4-1.0 folds and palmitic acid by 0.1-0.6 folds among the backcross lines when compared to the recurrent parents. Finally, the backcross lines with high oil (53-58\%), and low oil content (42-50\%) were selected for further evaluation [20]. Girnar 4 (ICGV 15083) and Girnar 5 (ICGV 15090) with about 80\% oleic acid content were approved for release and commercial cultivation in India (https://www.icrisat.org/indias-first-high-oleic-groundnutvarieties-ready-to-go-commercial/). Multi-season and site testing since 2014 have shown that the agronomic performance of the high oleic lines is significantly superior to the local popular varieties. ICGV 15090 and ICGV 15083 recorded 46\% and 42\% higher pod yield, respectively than the national check, TG 37A.

In another recent study, the mutant ahFAD2 alleles were transferred to GPBD 4 by MABC. The backcross lines were tested for the fatty acid composition under the glasshouse and field conditions. The oleic acid and linoleic acid contents ranged from 68.94-82.33\% and $1.74-10.87 \%$, respectively under glasshouse and from $67.04-$ $81.71 \%$ and $2.00-15.66 \%$, respectively, under field condition. The increase in the oleic acid content of the backcross lines over the recurrent parent varied from $28.78-53.80 \%$ and $33.70-62.96 \%$ under glasshouse and field conditions, respectively, indicating the stable expression of ahFAD2B in two different environments. On the contrary, linoleic acid showed $56.47-93.03 \%$ and $40.02-92.34 \%$ reduction among the backcross lines over the recurrent parent under glasshouse and field conditions, respectively [21]. Similar efforts are also being made to transfer the ahFAD2 alleles to G2 52, an improved variety at UAS, Dharwad, India.

TMV 2 is a farmer-friendly variety in Tamil Nadu, Andhra Pradesh and Karnataka. It was selected from Gudiyattam Bunch in 1940. It is very popular among the farmers because of its versatility and adaptation to diverse agroclimatic conditions. TMV 2 can profitably be grown during both rainy and post-rainy seasons. Excellent taste of the kernel and low oil content make TMV 2 a locally preferred confectionary variety. But TMV 2 is highly susceptible to late leaf spot (LLS) and rust diseases. Genomics-assisted breeding in TMV 2 resulted in the development of LLS and rust resistant backcross lines [22]. Now these backcross lines were employed for MABC with Sun Oleic 95R and its Spanish derivative ICGV 15025 to develop foliar disease resistant and high oleate genotypes with TMV 2 background [23].

With a goal to pyramid nematode resistance and high oleic: linoleic acid ratio in seeds of peanut, nematode-resistant cultivar Tifguard was used as the recurrent female parent and high $0 / \mathrm{L}$ cultivars Georgia-02C and Florida-07 were used as donor parents, and an improved genotype "Tifguard High O/L" was developed through MABC [24]. Inactivation of all $\delta 12$-desaturases in the developing seeds without effecting the gene expression in other tissues can be achieved by the implementation of transgenic technologies Oleic acid levels were increased to $85 \%$ in Brassica napus using antisense gene constructs [25], 85\% in soybean [26], and up to $89 \%$ in B. napus using co-suppression constructs [27]. Transgenics approach was also employed in peanut to enhance the oleic acid content. Sense and antisense silencing of ahFAD2 resulted in down-regulation of the FAD2, which led to increase in oleic acid content $(70 \%)$ in the seeds of transformed plants compared with a $37.93 \%$ in untransformed plants [28]. An RNAi vector for peanut FAD2 gene activated by its own promoter and containing its inverted repeat coding sequence fragments spliced by an intron was transformed into leaflet from germinating peanut seeds by the mediation of Agrobacterium in order to manipulate the content of oleic acid and linoleic acid in the seeds specifically. It was shown that the variation of oleic/linoleic ratio $(\mathrm{O} / \mathrm{L})$ within transgenic 
plants was greater than that within the wild type. Average O/L values of seeds in most transgenic lines were significantly higher than that of the wild type [29].

Recent studies on gene and genome-editing systems based on zinc finger nucleases, single-stranded oligonucleotides, TALE effector nucleases, and clustered regularly interspaced short palindromic repeats (CRISPR/Cas9) have been demonstrated in several polyploid crops for some or all of the genes targeted for modification on homologous chromosomes. These systems allow selection of desirable plants as null segregates that have lost their editing transgenes or even more attractively, by delivery of biodegradable Cas9/sgRNA ribonucleoprotein complexes (i.e., no DNA) into plant cells where they are expressed only transiently but allow for efficient gene editing. Such a system has been recently demonstrated Camelina sativa, a close relative of Arabidopsis thaliana and an emerging oil seed plant. Oleic acid content in the Camelina seeds was increased from 16\% to over $50 \%$. These increases were associated with significant decreases in the polyunsaturated fatty acids, linoleic acid (i.e. a decrease from $\sim 16 \%$ to $<4 \%$ ) and linolenic acid (a decrease from $\sim 35 \%$ to $<10 \%$ ) [30]. These innovative systems creating precise mutations can be useful in enhancing oleate content.

Overall, the genomics, transcriptomic and proteomics are taking forward the knowledge on the genetic control of oleic acid content in peanut. This knowledge would help in developing high oleate peanut genotypes to meet not only the nutritional requirements of a health-conscious society but also the industrial demands for better shelf life of oil and its products.

\section{References}

1. Grundy SM (1986) Comparison of monounsaturated fatty acids and carbohydrates for lowering plasma cholesterol. N Engl J Med 314(12): 745-748.

2. Toborek M, Lee YW, Garrido R, Kaiser S, Hennig B (2002) Unsaturated fatty acids selectively induce an inflammatory environment in human endothelial cells. The American Journal of Clinical Nutrition 75(1): 119125 .

3. Ahmed EM, Young CT (1982) Composition, quality, and flavor of peanuts. In: PHE \& YCT (Eds.), Peanut Science and Technology. American Peanut Research and Education Society Inc, Yoakum, Texas, USA, pp. 655-688.

4. Moore K, Knauft D (1989) The inheritance of high oleic acid in peanut. Journal of Heredity 80(3): 252-253.

5. Norden A, Smith O, Gorbet D (1982) Breeding of the cultivated peanut. In: Pattee, Young (Eds.), Peanut Science and Technology. American Peanut Research and Education Society Inc, Yoakum, Texas, USA, pp. 95-123.

6. Norden A, Gorbet D, Knauft D, Young C (1987) Variability in oil quality among peanut genotypes in the Florida breeding program. Peanut Science 14(1): 7-11.

7. Upadhyaya HD, Bramel PJ, Ortiz R, Singh S (2002) Developing a mini core of peanut for utilization of genetic resources. Crop Science 42(6): 21502156.

8. Mukri G, Nadaf HL, Bhat RS, Gowda, MVC, Upadhyaya HD, et al. (2012) Phenotypic and molecular dissection of ICRISAT mini core collection of peanuts (Arachis hypogaea L) for high oleic acid. Plant Breeding 131(3): 418-422.

9. Chu Y, Ramos L, Holbrook CC, Akins PO (2007) Frequency of a loss-offunction mutation in oleoyl-PC desaturase (ahFAD2A) in the mini-core of the US peanut germplasm collection. Crop Science 47(6): 2372-2378.

10.Holbrook CC, Dong W (2005) Development and evaluation of a mini core collection for the US peanut germplasm collection. Crop Science 45: 1540-1544.

11. Ashri A (1988) Mutagenic studies and breeding of peanut (Arachis hypogaea) and sesame (Sesame indicum). In: Improvement of grain legume production using induced mutations. International Atomic Energy Agency, Paris, France, pp. 509-511.

12.Gorbet D, Knauft D (1997) Registration of Sun Oleic 95R' peanut. Crop Science 37(4): 1392.

13. Gorbet D, Knauft D (2000) Registration of Sun Oleic 97R' peanut. Crop Science 40(4): 1190-1191.

14. Ray TK, Holly SP, Knauft DA, Abbott AG, Powell GL (1993) The primary defect in developing seed from the high oleate variety of peanut (Arachis hypogaea $L$ ) is the absence of $\Delta 12$-desaturase activity. Plant Science 91(1): 15-21.

15.Jung S, Powell G, Moore K, Abbott A (2000) The high oleate trait in the cultivated peanut [Arachis hypogaea L]. II. Molecular basis and genetics of the trait. Molecular and General Genetics 263(5): 806-811.

16.Wang Y, Zhang X, Zhao Y, Prakash C, He G, et al. (2015) Insights into the novel members of the FAD2 gene family involved in high-oleate fluxes in peanut. Genome 58(8): 375-383.

17. Nadaf HL, Biradar KS, Murthy GSS, Krishnaraj PU, Bhat RS, et al. (2017) Novel mutations in oleoyl-PC desaturase (ahFAD2B) identified from new high oleic mutants induced by gamma rays in peanut (Arachis hypogaea L). Crop Science 57(5): 2538-2546.

18. Chen Z, Wang M, Barkley N, Pittman R (2010) A simple allele-specific PCR assay for detecting FAD2 alleles in both A and B genomes of the cultivated peanut for high-oleate trait selection. Plant Molecular Biology Reporter 28(3): 542-548.

19.Wang C, Hu D, Ding F, Yu T, Tang Y, et al. (2011) A new set of allele-specific PCR primers for identification of true hybrids in normal oleatex high oleate crosses in groundnut. Journal of SAT Agricultural Research 9: 1-4.

20.Janila P, Pandey MK, Shasidhar Y, Variath MT, Sriswathi M, et al. (2016) Molecular breeding for introgression of fatty acid desaturase mutant alleles (ahFAD2A and ahFAD2B) enhances oil quality in high and low oil containing peanut genotypes. Plant Sci 242: 203-213.

21. Nawade B, Mishra GP, Radhakrishnan T, Sangh C, Dobariya J, et al. (2019) Development of high oleic peanut lines through marker-assisted introgression of mutant ahFAD2 alleles and its fatty acid profiles under open-field and controlled conditions. 3 Biotech 9(6): 243.

22.Kolekar RM, Sukruth M, Shirasawa K, Nadaf HL, Motagi BN, etal. (2017) Marker-assisted backcrossing to develop foliar disease resistant genotypes in TMV 2 variety of peanut (Arachis hypogaea L). Plant Breeding 136(6): 948-953.

23.Jadhav MP, Bhat RS, Pandey MK (2019) Marker-assisted breeding for high oleate content in groundnut. Paper presented at the National Conference on "Biodiversity and Plant Genetic Resource Conservation for Future", UAHS, Shivamogga, India.

24.Chu Y, Wu C, Holbrook C, Tillman B, Person G, et al. (2011) Markerassisted selection to pyramid nematode resistance and the high oleic trait in peanut. The Plant Genome 4(2): 110-117.

25. Topfer R, Martini N, Schell J (1995) Modification of plant lipid synthesis. Science 268(5211): 681-686.

26. Kinney AJ (1998) Plants as industrial chemical factories-new oils from genetically engineered soybeans. Lipid/Fett 100(4-5): 173-176.

27.Stoutjesdijk P, Hurlestone C, Singh S, Green A (2000) High-oleic acid Australian Brassica napus and B. juncea varieties produced by cosuppression of endogenous $\Delta 12$-desaturases. Biochemical Society Transactions 28(6): 938-940. 
28. Yin D, Deng S, Zhan K, Cui D (2007) High-oleic peanut oils produced by HpRNA-mediated gene silencing of oleate desaturase. Plant Molecular Biology Reporter 25(3-4): 154-163.

29. Huang B, Zhang X, Miao L, Yan Z, Hai Y, et al. (2008) RNAi transformation of Ah FAD2 gene and fatty acid analysis of transgenic seeds. Chin J Oil Crop Sci 30(3): 290-293.
30.Jiang WZ, Henry IM, Lynagh PG, Comai L, Cahoon EB, et al. (2017 Significant enhancement of fatty acid composition in seeds of the allohexaploid, Camelina sativa, using CRISPR/Cas9 gene editing. Plant Biotechnology Journal 15(5): 648-657.

For possible submissions Click below:

\section{Submit Article}

